\title{
How Age and Gender Influence the Performance of Mach Bands
}

\author{
Hongxuan Chen ${ }^{1, *, \dagger}$, Jingsheng Huang ${ }^{2,{ }^{,}, \dagger}$, Jing Zhang ${ }^{3, *, \dagger}$ \\ ${ }^{1}$ Tianjin No. 20 Middle School. Tianjin, China \\ ${ }^{2}$ Meihua School UIA, Kunshan, Jiangsu, China \\ ${ }^{3}$ Ashley Hall School, Charleston, SC, US \\ ${ }^{*}$ Corresponding author. Email: ${ }^{1} 2707911624 @ q q . c o m,{ }^{2} 1213664682 @ q q . c o m,{ }^{3}$ zhangj22@ashleyhall.org \\ These authors contributed equally.
}

\begin{abstract}
The purpose of this study is to investigate the influence of gender and age on the Mach band effect. 130 volunteers are recruited as volunteers to participate in our Mach Belt experiment. The results show that there is no significant difference between male and female students in the performance of Mach band effect, while the performance of minors was significantly better than that of adults. This research reveals the different effects of gender and age on Mach band phenomenon. Explanations for this discrepancy and implications for future research are discussed in the discussion section.
\end{abstract}

Keywords: Age, Gender, Mach Bands

\section{INTRODUCTION}

Mach bands is an optical illusion, due to the spatial high-boost filtering performed by the human visual system on the luminance channel of the image captured by the retina [1]. The physicist Ernst Mach first reported the effect in 1865 , conjecturing that filtering is performed in the retina itself through lateral inhibition among its neurons [2]. The Mach zone is a visual phenomenon caused by a lateral inhibitory impulse in the retina of the eye, which is recognized as a transparent or dense line due to the overlap of radiographic shadows in various situations. Since the Mach band is caused by the spatial high-gain filtering of the brightness channel of the image captured by the retina [3]. Hence, the difference of ophthalmic nerve between juveniles and adults may affect the Mach band strength.

As we all known, we can learn that in humans, eye formation begins at about three weeks of embryonic development and lasts until about ten weeks. The eye is formed from cells in the mesothelial and external tissues. Specifically, the eye is composed of neuroepithelium, surface ectoderm, and extracellular mesenchyma composed of the neural crest and mesoderm. The neuroepithelium forms the retina, ciliary body, iris, and optic nerve. The surface ectoderm forms the lens, corneal epithelium, and eyelid. The extracellular stroma forms the sclera, corneal endothelium and stroma, blood vessels, muscles, and vitreous [4].

\subsection{The role of age}

The pivotal difference between juvenile and adult brains is in the frontal lobe, which is made up of different amounts and types of cellular material and has neural characteristics different from that of the adult brain. The researchers connect the prefrontal cortex, a special part of the frontal lobe, with "mental flexibility, abstract thinking and the capacity to hold in mind related pieces of information" [5]. However, the frontal lobes from children cannot developed enough to perform these functions.

When people judge external circumstances, adolescents mostly used the amygdala (a small almondshaped region) for guiding instinctive responses, while adults rely on the frontal cortex to govern reason and planning [6]. Synapses are the mediums between the communication of neurons in the prefrontal cortex and neurons in other parts of the brain, and these synapses are significant in making judgments. For adults, this area of the brain is fully growing and connected to the other part of the brain, but to get to the level, it must go through a long period of chaotic development that begins in adolescence and lasts until the mid-20s. Additionally, the 
prefrontal cortex is less effectively functional in juveniles than that in adults. The neurons are partially covered by myelin sheath, which speeds up the transmission of information. This pad can increase transmission speed up to 100 times. But the myelin sheaths in teenage neurons are still semi-finished product [7].

\subsection{The role of gender}

The second independent variable is gender. As we know, Mach band phenomenon is a kind of vision illusion which full of three-primary colors, and some recent research show that there is a color Mach band. Certification of color Mach band proposed by P. L. Pease proposed. The color Mach band should be distinguished from three aspects, namely, the brightness Mach band, the saturation Mach band and the tonal Mach band, but it was not clear whether all the color Mach band existed under these three kinds of distinctions.

The research of Wang, Fu and Zhang shows that there is obvious Mach band effect in single-tone Mach pattern, and the light band and dark band of single-tone Mach band are quantitatively measured by the comparative matching method, and the variation of light band and dark band is measured. It provides a positive answer to the debate about whether there is a color Mach band [8]. Because of the certification of color Mach band, we can go further. The Mach band is a kind of illusion relative to color and vision. So, if we want to explore the feasibility of the experiment, we need to certificate the relationship between color and vision, as well as whether gender influence them or not. Research shows that color does affect vision. In the transmission of visual information, there are object shape and color visual illusion. The difference caused by the color contrast phenomenon is called color illusion, as a kind of visual phenomenon [9].

Finally, the present study needs to search about the influence of gender on color discrimination. Analysis of the experimental data of Huang, Liang, Yao and Zhu, showed that women were better at color recognition than men, while male observers were less able to recognize certain colors, such as orange red and turquoise [10]. The light source has a great effect on the observer's perception of color differences. In visual attributes, males are better in visual activities contrast and sports, but female just better in color [11]. I use literature Review Method to affirm the occurrence of the above phenomenon. As the result, gender influence the color discrimination and visual, and the Mach band also depend on them. So, theoretically, sex influences the Mach band phenomenon.

\subsection{The current study}

The first purpose of our study is to further explore the relationship between demographic variables and Mach band phenomenon, which is rarely involved before, so as to find out the influence of color and light on these variables as much as possible. Second, we hope that through our research, Mach band can bring more help and inspiration to people in the field of painting. For example, for people of different genders and ages, what kind of paintings can give people a greater visual impact, or make them prefer. We suspect that women are more sensitive to color, and may have an advantage in the color Mach band phenomenon, but may be less so in the trichromatic type of experiment. In medicine, since the development of the optic nerve with age is related to the Mach band effect, doctors can use the Mach band effect to perform simple eye check-up for people. We suspect that adults may have higher accuracy in the Mach band effect than adolescents.

\section{METHOD}

\subsection{Participants}

We use mathematical statistics method, Questionnaire method and Literature Review Method to done our testing. Our questionnaire collected answers from 130 volunteers. And got a proper gender ratio [male $(\mathrm{M}=54.71 \%, \quad \mathrm{SD}=0.19)$ and female $(\mathrm{M}=51.83 \%$, $\mathrm{SD}=0.19)]$ as well as enough number of test-takers of all age adult $(\mathrm{t}=2.885, \mathrm{p}=0.005<0.05)$ minors $(\mathrm{M}=60.81$, $\mathrm{SD}=0.20$ ), which helped us a lot when we studied the influence of age and gender.

\subsection{Experimental process}

We use the app called" Questionnaire Stars" to design 10 questions, and try to find out the regularities of Mach band through them. The following is our detailed experimental procedures. First, we record basic information of subjects. Then the subjects were asked to answer questions on a questionnaire (which included images of different kinds of Mach band phenomena). Finally, we use SPSS to analyze the results and draw a conclusion.

\section{RESULTS}

After the correlation analysis of the core variables in this study, it was found that whether adulthood was significantly related to Mach band achievement. 
Table 1. Descriptive statistics and correlation analysis tables $(\mathrm{N}=130)$

\begin{tabular}{|c|c|c|c|c|}
\hline & Average $(\mathrm{M} \pm \mathrm{SD})$ & Gender & Adult & $\begin{array}{l}\text { Mach band } \\
\text { Performance }\end{array}$ \\
\hline Gender & $1.539 \pm 0.50$ & 1 & & \\
\hline Adult & $1.285 \pm 0.45$ & $-0.271^{* *}$ & 1 & \\
\hline Mach band Performance & $0.534 \pm 0.190$ & 0.076 & $0.247^{\star *}$ & 1 \\
\hline
\end{tabular}

Note: sex $1=$ female $2=$ male; adult $1=$ adult $2=$ minor. ${ }^{*} * \mathrm{p}<0.01$.

The results showed that there was no significant difference between male ( $\mathrm{M}=54.71 \%, \mathrm{SD}=0.19)$ and female $(\mathrm{M}=51.83 \%, \mathrm{SD}=0.19)$ in the overall Mach band task $(\mathrm{M}=0.860,0.392>0.05)$. There was a significant difference in whether to adult $(\mathrm{t}=2.885, \mathrm{p}=0.005<0.05)$. The correct rate of minors $(\mathrm{M}=60.81, \mathrm{SD}=0.20)$ was significantly higher than that of adults $(M=50.43$ $\mathrm{SD}=0.18)$.

Further, in this study, gender, whether adulthood was used as an inter-subject factor. a two-factor analysis of variance of Mach band scores found that the main effect margin of gender was significant $(F(1,126)=2.809$, $\mathrm{p}=0.096)$, and whether the main effect of adulthood was significant $(\mathrm{F}(1,126)=10.597, \mathrm{p}=0.001)$, while the interaction between the two was not significant.

Combined with the results of $t$ test and ANOVA, it can be seen whether adulthood has a significant effect on Mach band achievement and minors perform better, and the gender factors of the subjects may also influence Mach band. However, this gender difference is only reflected in the edge of the main effect of ANOVA. Specifically, the correct rate of men will be slightly higher than that of women.

\section{DISCUSSION}

\subsection{Certain nerve cells in the brain \& age}

A sample of Chinese people of different ages participated in this study, the data were collected through self-reports. Results revealed that participants' Mach band effect becomes more pronounced as the optic nerve develops [11]. The optic nerve, also known as the second cranial nerve, is composed of axons that transmit visual information from the neurosensory retina to the visual cortex [12].

Adults and minors in the sensitivity of color there is an obvious gap. Since global reduction of hemispheric laterality was identified with a worse decline in parietal and occipital lobes as age advances. Reservation and leftright shift of laterality were vastly detected in the highlevel cognition-related regions, which may imply a compensatory mechanism of healthy [11] brain counteracting the age-associated functional impairment. The age dependence on the hemispheric asymmetry may embed mechanisms that underlie the [13] behavioral and cognitive declination associated with age related neuropsychological diseases. It's inevitable that the sensitivity to light will decrease under the impact of age
[12]. Therefore, with the increasing of age, the stimulation of receiving light will be reduced. The lower the degree of excitement, the lower the subjective perception of brightness.

\subsection{Gender}

Through our exam we think in the Mach band phenomenon, different genders react differently to different color combinations and arrangements, which means men and women react differently to the Mach band phenomenon and focus their observations differently. And these situations maybe relative to the "Visual Motion Perception' and the 'Color sensitivity" Contrast sensitivity' and 'Visual acuity'.

Through the literature, the women are better in 'Color sensitivity', which refers to the human ability to perceive colors. In color perception, one of the obvious gender differences is the male color vision deficiency [18-13], that means when different colors are mixed, females are more likely to recognize the colors and be more sensitive to the differences. What's more Contrast sensitivity is the ability of a person or animal to sense changes in brightness (Exam with intersecting rectangles/ stripe patterns). Females are more sensitive at lower spatial frequencies, while males are more sensitive at higher spatial frequencies [14]. These results can explain the consequences of stripe patterns in our exam, and because of the gender data in Contrast sensitivity can't shows an exact answer, and females are more likely to recognize the colors and be more sensitive to the differences, so in the test like colorful intersecting rectangles, women done better.

The 'Visual Motion Perception' in the same research [15], shows that the threshold of motion perception was higher in women than in men, indicating that women were weaker than men in their ability to integrate local information into global motion directions [16]. The exam through their research used a picture with some three primary colors intersecting rectangles and a line as a test subject, which is same as the pictures we used in our test. And that's prove the result 'Males are better deal with the kind of three primary colors intersecting rectangles in Mach band'. 'Visual acuity' is the ability of the eye to distinguish objects in detail, and the researchers found that men performed better on visual acuity tasks [17]. Therefore, in the real pictures composed of three primary colors, men's sensitivity to details and accuracy of the problem are higher than women. Through our testing, in 
the Mach band, men were more sensitive to the light and shade of the trichromatic rectangles, as well as to the images in which colors were wrapped around each other. Women were more sensitive to images with three primary colors wrapped around each other, and to the light and shade of colored rectangles.

\subsection{Hobbies}

There is another assumption about the hobbies make an influence on Mach band. As we all know, the cerebral cortex has four regions--- $\mathrm{V} / \mathrm{H} / \mathrm{S} / \mathrm{W}$. And the $\mathrm{V}$ region, control about our vision. We guess that different hobbies can develop different regions. And the one which can develop $\mathrm{V}$ region, can make people get a better result in Mach band test.

Through all of our feedbacks, we can divide them into four types: Sport, Art, Technology and Leisure. Research shows that physical exercise and keeping a good mood are beneficial to maintaining visual health [18]. The visual cortex is the highest center of vision. The visual information is transmitted to the visual cortex through the visual pathway, and then the visual perception is generated through the analysis and synthesis of the visual cortex. So, if exercise (sport) really can be benefit to the vision, it must be developed the visual cortex ( $\mathrm{V}$ region), as a result pay off the Mach band. In addition, some of the hobbies such as technology, also let their people get a brilliant result in the test, and one of them even totally correct in 10 questions. But all these are just a guess, and there are no paper can prove these assumptions are true.

\subsection{Limitations and future directions}

There are some limitations for the experiment is that maybe people don't show their subjective feelings for the sake of accuracy. And the effect of myopia on Mach band response, the kind of visual illusion testing---Mach band might be influence by whether people are wearing glasses or not. What's more, limitations in the selection of subjects and uneven age distribution. Moreover, there are limitations in the question raising, with a few questions and uneven distribution of different types. Gender differences may not be so big. The evidence for this study is outlined in the introduction, which itself may be less reliable. In addition, the differences between men and women are very small in conclusion. But in fact, there is a gender difference, they just don't see it. However, the evidence from this study is solid and encourages further verification by other researchers in the future

According to our study, Mach band phenomenon is less involved in demographic variables, in other words, it still has a lot of room for development. Through the investigation of gender and age, this experiment simply obtained the sensitivity difference of different demographic variables to Mach band and the different responses. This result can be widely used in the painting industry, and even in the decoration industry, through people's illusion of color -- side inhibition phenomenon (Mach band), so that the product becomes more impact color, so that customers can buy more cost-effective, more comfortable products.

Due to the influence of background density on specific structures, a similar phenomenon usually occurs in computed tomography, that is, the background contrast effect. This may be the source of the diagnosis error. To avoid this problem, a computer should be used instead of just relying on the observer's eyes to measure the density of related structures [19]. The influence of the Mach zone on art can be traced back at least to the Tang Dynasty (618 to 907) in China, Asia. In the European Middle Ages, the use of Mach bands and, in fact, the use of shadows, perspective drawings and other realist elements has largely disappeared. During the Renaissance, the spirit of scientific inquiry and the artistic means of depicting a more realistic and perceptual world reappeared. Brunelleschi and Alberti began to popularize their vision in the early 15 th century, and the portrayal of shadows reappeared. The Mach band quickly followed up. Painters like Montegna and Leonardo painted light bands on the edges of dark borders, and vice versa [20]. "we should place a light background in contrast to a shadow and a dark background to a mass of light, which is a general principle, a precept not to be attacked" , Leonardo da Vinci said [14]." Mach bands not only influence the art, but also the medicine which affect all of us. An example is the radiolucent halo that is sometimes seen around breast cancers [14]. Also, pseudopneumothorax secondary to skin fold is another example [21]. Due to this fact, Mach Band is very important.

\subsection{The importance of the experiment}

Due to the influence of background density on specific structures, a similar phenomenon usually occurs in computed tomography, that is, the background contrast effect. This may be the source of the diagnosis error. To avoid this problem, a computer should be used instead of just relying on the observer's eyes to measure the density of related structures [3]. The influence of the Mach zone on art can be traced back at least to the Tang Dynasty (618 to 907) in China, Asia. In the European Middle Ages, the use of Mach bands and, in fact, the use of shadows, perspective drawings and other realist elements has largely disappeared. During the Renaissance, the spirit of scientific inquiry and the artistic means of depicting a more realistic and perceptual world reappeared Brunelleschi and Alberti began to popularize their vision in the early 15th century, and the portrayal of shadows reappeared. The Mach band quickly followed up. Painters like Montegna and Leonardo painted light bands on the edges of dark borders, and vice versa [4]. "Leonardo da Vinci says we should place a light background in contrast 
to a shadow and a dark background to a mass of light. And it is a general principle, a precept not to be attacked [5]." Mach bands not only influence the art, but also the medicine which affect all of us. An example is the radiolucent halo that is sometimes seen around breast cancers [6]. Also, pseudopneumothorax secondary to skin fold is another example [7]. Due to this fact, Mach Band is very important.

\section{CONCLUSION}

The whole study focuses on demographic variables to study the Mach band phenomenon. Under the interference of limitations, this study believes that men and women have almost the same sensitivity and reaction results in the Mach band phenomenon, which is quite contrary to the theory. The surprising conclusion of the age group is that juveniles consistently perform far better than adults in Mach band phenomenon, which is also very conducive to other researchers to carry out more indepth studies.

\section{AUTHORS' CONTRIBUTIONS}

All authors contributed to this article equally. The part responsible for each person: Hongxuan Chen, section of 2, 4.2, 4.3, 4.4, and conclusion; Jingsheng Huang, section of abstract and 4.1; Jing Zhang, section of introduction and 4.5; Hongxuan Chen, Jingsheng Huang, and Jing Zhang, introduction; Hongxuan Chen, Jingsheng Huang and Jing Zhang, section of results.

\section{REFERENCES}

[1] Daffner $\mathrm{R} H$. Visual illusions in computed tomography: phenomena related to Mach effect[J]. American Journal of Roentgenology, 1980, 134(2): 261-264. DOI: 10.2214/ajr.134.2.261

[2] Gordenne W H, Malchair F L. Mach bands in mammography[J]. Radiology, 1988, 169(1): 55-58. Doi: 10.1148/radiology.169.1.2843941

[3] Jampel R S. Mach bands: Quantitative studies on neural networks in the retina[J]. Vision Research, 1966, 6(7):484-484. DOI: 10.1016/00426989(66)90057-5

[4] Dr Yogesh Sontakke, MBBS MD (Anatomy) Jawaharlal Institute of Postgraduate Medical Education and Research (JIPMER), from http://www.eophtha.com/posts/embryology-of-eye

[5] Carlén M. What constitutes the prefrontal cortex? [J]. Science, 2017, 358(6362): 478-482. DOI: $10.1126 /$ science.aan 8868

[6] Weaterton R, Tan S, Adam J, et al. Beyond Visual Acuity: Development of a Simple Test of the SlowTo-See Phenomenon in Children with Infantile
Nystagmus Syndrome[J]. Current Eye Research, 2021, 46(2): 263-270. DOI: $10.1080 / 02713683.2020 .1784438$

[7] Wang Z, Fu Y, Zhang Q. Research on the phenomenon of color illusion. Department of Physics, Sichuan University.

[8] What's the difference between teenage brains and adult brains? Updated On: 1 Feb 2021 By Vaishnavi Patil, from Science ABC

[9] Wang Z, Xu H. Study on color visual discrimination characteristics and color aberration evaluation. Zhejiang University, 2009.

[10] Hao H, Jing L, Yao B, \& Zhu G. Gender Effects on Color Discrimination. Dalian Polytechnic University.

[11] Tong N, Kuang S. Sex differences in the visual system. Department of Psychology, University of Chinese Academy of Sciences.

[12] Hind A, Daniah A, \& Tariq A. Optic Nerve: Developmental Anomalies and Common Tumors, Optic Nerve, $2018 . \quad$ DOI: 10.5772/intechopen.80326.Available from: https://www.intechopen.com/books/opticnerve/optic-nerve-developmental-anomalies-andcommon-tumors

[13] Ye W, Chen Z, Tang P, Song X, \& Dai S. Sex difference and analysis of human visual response time. Psychological Science, 1998(1): 74-74. DOI: CNKI:SUN:XLKX.0.1998-01-017

[14] Abramov I, Gordon J, Feldman O, et al. Sex \& vision I: Spatiotemporal resolution. Biology of Sex Differences, 2012, 3(1): 20-20

[15] Billino J, Bremmer F, Gegenfurtner $\mathrm{K}$ R. Differential aging of motion processing mechanisms: evidence against general perceptual decline. Vision Res, 2008, 48(10): 1254-1261. DOI: $10.1016 /$ j.visres.2008.02.014

[16] Ishigaki H, Miyao M. Implications for dynamic visual acuity with changes in age and sex. Perceptual and Motor Skills, 1994, 78(2): 363-369.

[17] Ong S R, Crowston J G, Loprinzi P D, et al. Physical activity, visual impairment, and eye disease[J]. Eye, 2018, 32(8): 1296-1303. DOI: 10.1038/s41433-0180081-8

[18] Homer W I. Seurat and the Science of Painting[J]. 1964.

[19] Kishimoto K, Watari T, Tokuda Y. Pseudopneumothorax: skin fold is an excellent imitator[J]. Case Reports, 2018, 2018: bcr-2018-226360. Doi: $10.1136 /$ bcr-2018-226360 
[20] Khosravy M, Gupta N, Marina N, et al. Perceptual adaptation of image based on Chevreul-Mach bands visual phenomenon[J]. IEEE Signal Processing Letters, 2017, 24(5): 594-598. DOI: 10.1109/LSP.2017.2679608

[21] Marmor M F. Mach Bands and Art[J]. Basic and Clinical Applications of Vision Science, 1997. 